\section{Onset of Schamberg Disease and Resolution of Alopecia Areata During Treatment of Atopic Dermatitis With Dupilumab}

Szekely $\mathrm{S}^{1}$, Vaccari D ${ }^{1}$, Salmaso R ${ }^{2}$, Belloni-Fortina A ${ }^{1}$, Alaibac $\mathrm{M}^{1}$ ${ }^{1}$ Unit of Dermatology, University of Padua, Padua, Italy

${ }^{2}$ Unit of Pathology, University of Padua, Padua, Italy

J Investig Allergol Clin Immunol 2021; Vol. 31(1): 65-66 doi: 10.18176/jiaci.0541

Key words: Schamberg disease. Alopecia areata. Dupilumab. Atopic dermatitis.

Palabras clave: Enfermedad de Schamberg. Alopecia areata. Dupilumab. Dermatitis atópica.

Atopic dermatitis (AD) is a chronic pruritic immunemediated inflammatory dermatosis with a high prevalence both in children and in adults. Its pathogenesis is multifactorial, including genetic, immunological, and environmental factors that cause skin barrier dysfunction, alterations in cell-mediated immune responses, and IgE-mediated hypersensitivity. Dupilumab is an interleukin 4 (IL-4) receptor $\alpha$ antagonist that inhibits IL-4/IL-13 signaling through blockade of the shared receptor subunit for IL- $4 \alpha$. This in turn leads to downregulation of the $\mathrm{T}_{\mathrm{H}} 2$ immune response, which is the mechanism responsible for the efficacy of dupilumab in patients with AD [1]. Schamberg disease, also known as progressive pigmented purpuric dermatitis, is the most common pigmented purpuric dermatosis. It is a recurrent skin disorder characterized by nonpalpable symmetrical pinpoint petechial and pigmented macules, purpura, and, sometimes, telangiectasia, especially on the extremities [2]. The etiology is unknown, although immunemediated mechanisms may play a role. Alopecia areata (AA) is an autoimmune nonscarring alopecia with heterogeneous severity that affects up to $2 \%$ of the general population. Currently available treatment options for AA are of limited efficacy and have been associated with adverse effects [3].

Here, we report the case of a patient affected by severe $\mathrm{AD}$ and $\mathrm{AA}$ on the scalp who developed a form of pigmented purpuric dermatosis, with concomitant improvement of AA during treatment with dupilumab for AD.

A 30-year-old man with AD since childhood was referred to our unit. Physical examination revealed severe erythema, thickness, and lichenification affecting his entire body (Eczema Area and Severity Index [EASI], 36; Dermatology Life Quality Index [DLQI], 18).

He had previously undergone several conventional treatments for $\mathrm{AD}$, including systemic corticosteroids, phototherapy, methotrexate, and cyclosporine, which was discontinued because of nephrotoxicity. The patient also had a 2-year history of AA affecting the entire surface of the scalp, with sparing of the eyebrows and beard. He had received topical and systemic corticosteroids, with no significant improvement.

Owing to intolerance and failure of the previous therapies, dupilumab was started as a treatment for $\mathrm{AD}$ at an initial dose of $600 \mathrm{mg}$ and subsequent 300-mg injections every 2 weeks. After 3 months of follow-up, clinical examination revealed an improvement in AD (EASI, 9.6; DLQI, 8) and onset of numerous 2- to 3-mm reddish brown, nonblanching, petechial nonpruritic macules scattered on both forearms and dorsa of the hands (Figure, A). He denied itching and burning. Punch biopsy of a purpuric lesion revealed hyperkeratosis and acanthosis of the epidermis, mild dermal perivascular lymphocytic infiltration, and erythrocyte extravasation and hemosiderin deposition consistent with Schamberg disease. Direct immunofluorescence was negative for perivascular and dermoepidermal immunoglobulin and complement deposition. Dupilumab was discontinued because of this adverse effect. The patient's hair had regrown completely 3 months after

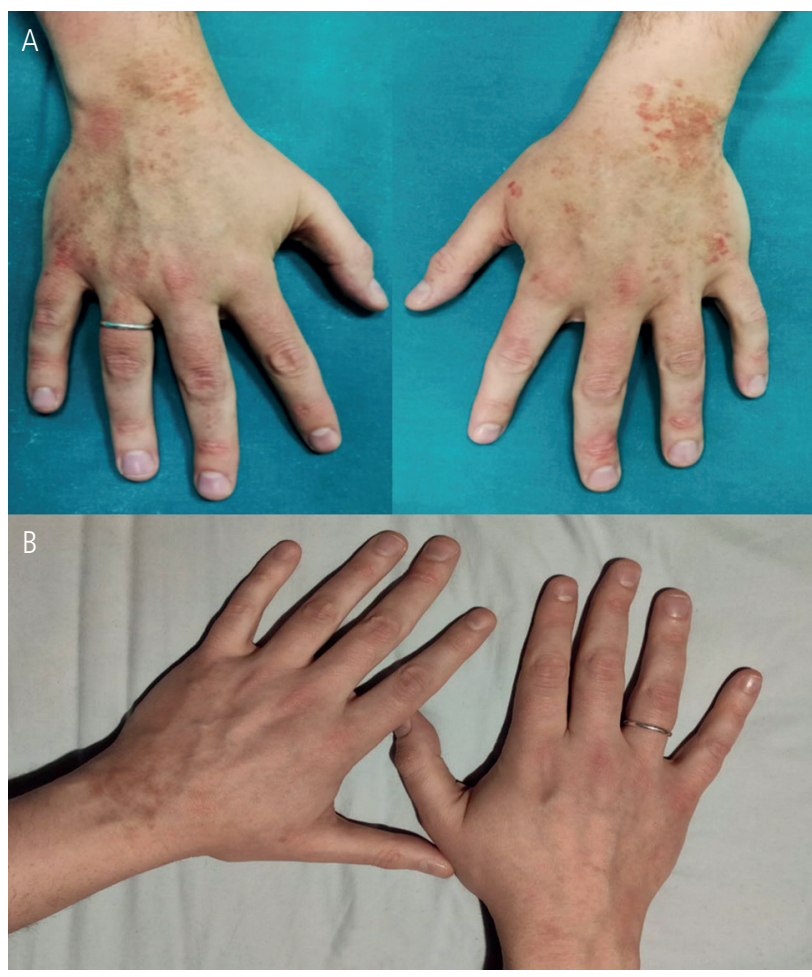

Figure. A, Reddish brown, petechial, nonpruritic macules scattered on both forearms and the dorsa of the hands. The histological examination was consistent with a diagnosis of Schamberg disease. B, Almost complete remission of the lesions after discontinuation of dupilumab. 
discontinuation, with no recurrence of AA at follow-up visits 1,3 , and 6 months after discontinuation and progressively complete remission of Schamberg disease (Figure, B). There were no recurrences of AD (EASI, 5,2; DLQI, 5).

Schamberg disease is a member of the group of pigmented purpuric dermatoses, which include a spectrum of vascular diseases with various clinical manifestations, but with some common histopathological features, including epidermal changes (hyperkeratosis, parakeratosis, and acanthosis), perivascular lymphocytic infiltration, red blood cell extravasation, hemosiderin deposition, endothelial cell swelling, spongiosis, lymphocyte exocytosis, and lichenoid lymphocytic infiltration [4]. Vascular damage and erythrocyte leakage are probably secondary to a localized T cell-mediated reaction in the vicinity of the dermal capillaries. Risk factors include venous hypertension, stasis, exercise, trauma, contact allergy, dietary factors, alcohol intake, and systemic and focal infections. Drug-induced cases of Schamberg disease have been reported with aspirin, thiamine, acetaminophen, and amlodipine [4].

Dupilumab is the first biologic drug approved for the treatment of moderate-to-severe AD. Inhibition of the release of proinflammatory cytokines, chemokines, and IgE make this monoclonal antibody suitable for both $\mathrm{AD}$ and $\mathrm{AA}$, which share some $\mathrm{T}_{\mathrm{H}} 2$-mediated pathogenetic mechanisms [1]. Although dupilumab is considered a safe drug, high rates of unspecified conjunctivitis have been reported [1]. Other adverse effects include injection site reactions, exacerbations of $\mathrm{AD}$, headache, skin infections (bacterial and herpetic), nasopharyngitis, and headache [1]. The association between dupilumab and Schamberg disease has never been described in clinical trials or case reports. Nevertheless, the present case does not allow us to draw definitive conclusions concerning the role of dupilumab in Schamberg disease, given that readministration of the drug was not considered in order to avoid triggering an eventual relapse.

AA can be associated with atopy, although the relationship between dupilumab and AA is controversial: some reports describe dupilumab-induced alopecia [5-7], whereas others show improvement in AA related to the treatment $[8,9]$. AA and AD share a similar genetic background-both diseases commonly co-occur-and other, shared clinical and immunological pathways and features, in particular a strong $\mathrm{T}_{\mathrm{H}} 2$ component and up-regulation of IL-23 [5,10]. Up-regulation of $\mathrm{T}_{\mathrm{H}} 2$ pathway genes is common in both $\mathrm{AD}$ and AA; consequently, down-regulation with dupilumab can explain the therapeutic efficacy in AA [10]. However, other immunological mechanisms might amplify the $T_{H} 1$ pathway and promote the development of AA after treatment with dupilumab [10]. According to previous reports, dupilumab can induce regrowth of hair in AA, usually 3-6 months after initiation of therapy, although it may begin as soon as after the initial doses $[8,9]$. In the case we report, AA resolved completely after 3 months of therapy.

In conclusion, this is the first case of a patient with severe $\mathrm{AD}$ and $\mathrm{AA}$ treated with a short course of dupilumab showing both development of Schamberg disease and simultaneous complete resolution of AA.

\section{Funding}

The authors declare that no funding was received for the present study.

\section{Conflicts of Interest}

The authors declare that they have no conflicts of interest.

\section{References}

1. Sastre J, Dávila I. Dupilumab: A New Paradigm for the Treatment of Allergic Diseases. J Investig Allergol Clin Immunol. 2018;28:139-50.

2. Kutlubay Z, Cesur SK, Aşkın Ö, Tüzün Y. The color of skin: brown diseases of the skin, nails, and mucosa. Clin Dermatol. 2019;37:487-506.

3. Gilhar A, Etzioni A, Paus R. Alopecia areata. N Engl J Med. 2012;366:1515-25.

4. Sardana K, Sarkar R, Sehgal VN. Pigmented purpuric dermatoses: an overview. Int J Dermatol. 2004;43:482-8.

5. Flanagan K, Sperling L, Lin J. Drug-induced alopecia after dupilumab therapy. JAAD Case Rep. 2019;5:54-6.

6. Barroso-García B, Rial MJ, Molina A, Sastre J. Alopecia areata in severe atopic dermatitis treated with dupilumab. J Investig Allergol Clin Immunol. 2018;28:420-1.

7. Ständer S, Trense Y, Thaçi D, Ludwig RJ. Alopecia areata development in atopic dermatitis patients treated with dupilumab. J Eur Acad Dermatol Venereol. 2020; 34(10):e61213.

8. Smogorzewski J, Sierro T, Compoginis G, Kim G. Remission of alopecia universalis in a patient with atopic dermatitis treated with dupilumab. JAAD Case Rep. 2019;5:116-7.

9. Alniemi DT, McGevna L. Dupilumab treatment for atopic dermatitis leading to unexpected treatment for alopecia universalis. JAAD Case Rep. 2019;5:111-2.

10. Kridin K, Renert-Yuval Y, Guttman-Yassky E, Cohen AD. Alopecia areata is associated with atopic diathesis: results from a population-based study of 51,561 patients. J Allergy Clin Immunol Pract. 2020;8:1323-8.

Manuscript received April 11, 2020; accepted for publication May 25, 2020.

Mauro Alaibac

Unit of Dermatology University of Padua Via Gallucci 4

35128 Padua, Italy E-mail: mauro.alaibac@unipd.it 